\title{
Synthesis of Biodegradable Polyolefins Based Polymer Composites Using Degradable Natural Materials
}

\author{
Samarasekara A.M.P.B ${ }^{*}$ and Jayasuriya E.A.P.C.D \\ Department of Materials Science and Engineering, University of Moratuwa, Katubedda, Sri Lanka \\ banduamp@yahoo.com
}

\begin{abstract}
Polymers are high molecular mass compounds and their structure is composed of a large number of repeating units. These days, polymer materials are so important in our daily life that survival of human life depends on these polymers. Polymer materials have wide range of properties that canbe used in different applications. There has been increasing public concern over the harmful effects of petrolium-based polymer packaging materials specially polyolefins in the environment after the usage. These pectrolium - based polymers create significant amount of waste after the usage and that generates the critical environmental issues.Recycling, recovery and disposal of plastic waste are some of the options available. These methods have certain disadvantages and not hundred percent practical. An important alternative to minimize the polymer waste is the introduction of biodegradable polymers, which can be degraded through the action of naturally occurring microorganisms.
\end{abstract}

The objective of this research was to produce biodegradable composite material with the help of Low Density Polyethylene (LDPE), cellulose - hemi cellulose and natural enzymes. Cellulose and hemi cellulose are two types of biodegradable natural polymers. Cellulose hemi cellulose mixtures were extracted using rice straw which is highly available in Sri Lanka as an agricultural waste. Approximately $33 \%$ of cellulose - hemi cellulose mixture was extracted using rice straw. The biodegradability impartedby cellulose - hemi cellulose can be further enhanced by introducing natural enzymes. Radish peroxidase is one of the natural enzymes that were used to enhance the biodegradability of cellulose - hemi cellulose. Radish peroxidase was extracted by using radish roots.The extracted cellulose - hemi cellulose and radish peroxidase were analysed and characterized using Fourier Transform Infrared (FTIR) Spectroscopy. Main Functional groups related to cellulose - hemi cellulose and radish peroxidase were available in the extracted FTIR spectra. LDPE - cellulose - hemi cellulose radish peroxidase polymer blend was prepared using laboratory scale internal mixture by using $1 \mathrm{wt} \%$ cellulose - hemi cellulose and varying $0.5 \mathrm{wt} \%$ to $2.5 \mathrm{wt} \%$ radish peroxidase. The mixing temperature and screw speed were $150{ }^{\circ} \mathrm{C}$ and $60 \mathrm{rpm}$ respectively. Hydraulic press was used to prepare the biodegradable polymer composite material. The degree of biodegradability was evaluated by measuring time dependent weight loss, water absorption and tensile properties of the developed composite. Soil burial test was used to measure the time dependent weight loss. Pure LDPE samples showed the high strength and constant weight during the soil burial test without degradation. Samples containing cellulose - hemi cellulose - radish peroxidase showed a significant degradation during the testing period. According to the experimental results, this composite material can be used for various applications and their development is a good option for reducing the consumption of petroleum resources and environmental pollution issues.

Keywords: Radish peroxidase, Biodegradable, Cellulose - hemi cellulose, Polymer 